\title{
DYNAMIC STEREOSCOPIC PREVIZ
}

\author{
Sergi Pujades ${ }^{1}$, Laurent Boiron ${ }^{2}$, Rémi Ronfard ${ }^{2}$, Frédéric Devernay ${ }^{1}$ \\ ${ }^{1}$ Laboratoire d'Informatique de Grenoble, Univ. Grenoble Alpes \& Inria, France. \\ ${ }^{2}$ Laboratoire Jean Kuntzmann, Univ. Grenoble Alpes \& Inria, France.
}

\begin{abstract}
The pre-production stage in a film workflow is important to save time during production. To be useful in stereoscopic 3-D movie-making, storyboards and previz tools need to be adapted in at least two ways. First, it should be possible to specify the desired depth values with suitable and intuitive user interfaces. Second, it should be possible to preview the stereoscopic movie with a suitable screen size. In this paper, we describe a novel technique for simulating a cinema projection room with arbitrary dimensions in a realtime game engine, while controling the camera interaxial and convergence parameters with a gamepad controller. Our technique has been implemented in the Blender Game Engine and tested during the shooting of a short movie. Qualitative experimental results show that our technique overcomes the limitations of previous work in stereoscopic previz and can usefully complement traditional storyboards during pre-production of stereoscopic 3-D movies.
\end{abstract}

Index Terms - 3D video capture and 3D-TV, Scene modelling, Pre-visualization.

\section{INTRODUCTION}

Stereoscopic movie-making process is a complex task involving mainly two stages: the acquisition and the projection. In the acquisition stage, two cameras are mounted in a stereoscopic rig. The rig allows to control the distance between the cameras (interaxial) and their relative angle. This angle will define the convergence distance. In the projection stage, the two acquired images are projected onto the same screen for a spectator to watch them.

The director and the stereographer face an important question: how to set the acquisition parameters e.g., interaxial and convergence distance, in order to obtain the desired 3D effect in the projection room. This choice is difficult because the relationship between the 3D of the acquired scene and the perceived 3D in the projection room is complex. Moreover, 3D perception during the projection stage includes physiological constraints thay may cause visual fatigue [13], due to, e.g., ocular divergence or convergence/focus dissociation.

In recent years, expert directors and stereoographers have proposed some useful rules of thumb that can be used to over- come these difficulties, by containing the acquisition parameters in a "3D safe zone". For example the 1/30th rule states that "the interaxial distance should be 1/30th of the distance from the camera to the first foreground object" [8]. This rule is very handy for safe filming, but very limiting in terms of 3D creativity. In order to create novel 3D narratives [9], some "not-so-safe" configurations should also be explored.

In order to explore and test new configurations, working with actual equipment is cumbersome. Creating an actual shooting set is time consuming and expensive, involving often important human resources. Instead of using the actual acquisition devices (cameras, rigs, actors, sets,...) and different projection rooms (tv, $10 \mathrm{~m}, 20 \mathrm{~m}$ screens) we propose to work in a virtual environment: the movie pre-visualization (previz). Previz consists of a virtual environment allowing the director to literally "see his film before shooting it" [11] by creating simplified 3D models of the scene, and generating computer-generated versions of the movie shot by shot. It is widely used in the pre-production phase of film-making. It gives directors the opportunity to easily explore novel and original shooting configurations, without the high cost of an actual shooting test. Moreover, previz can also be very helpful for teaching movie-making, as students can interactively play with camera parameters and see the effects of their choices.

Existing previz tools adressed a subset of these stereoscopic shooting problems. We built on them to provide a new previz tool that supports two crucial characteristics:

Real-time dynamic manipulation of the scene and camera controls with a physical device: existing tools are either static or provide an animation based on keyframes, that are automatically interpolated. However an operator would like to rehearse as if he was on-set. In particular, dynamic shots are difficult to master. For example, panning shots and dolly shots are very frequent in modern movie-making, and require a lot of preparation. Because the image content varies dramatically during such shots, it is particularly important to control stereoscopic parameters dynamically. Yet, existing previz tools offer limited control of interaxial and convergence distance dynamics. As a result, some directors and stereographers tend to adopt conservative values that can be kept constant for the entire shot, even in previz. Real-time control of camera interaxial and convergence distance is needed to rehearse this more complex shots in previz. 
Depth information visualization: the transformation between the real 3D in the scene and the perceived 3D in the projection room is complex. Small changes in the acquisition stereoscopic parameters can significantly modify the perceived 3D effect in the projection room. It is highly difficult to visually understand how this transformation deforms the 3D objects. The main issue is that the size of the screen used for projection will influence the perceived 3D [1]. For example, it is not possible to pre-visualize $3 \mathrm{D}$ on a laptop screen if the target is a movie theater with a $10 \mathrm{~m}$ wide screen. Other existing methods, based on meta-data feedback (e.g., depth-budget [4, 15]) are not easy to read and lack deformation information.

In this paper, we present a Dynamic Stereoscopic Previz (DSP) system which overcomes the above limitations. On the one hand, DSP allows a real-time, direct interaction in a game engine, which makes it suitable for use by directors, cinematographers and stereographers. In single user mode, DSP allows camera parameters to be recorded one by one, within a dedicated multi-track recording system. In multi-user mode, DSP allows camera parameters to be recorded from multiple devices. On the other hand, we propose a novel method for simulating a virtual projection room (Sec. (4) that allows seeing, not only a volume, but also the arrangement of the 3D elements of the scene as they will be perceived in the specified projection room. One important property of this view is that it scales with the target screen size. Moreover the representation gives more relevant information than the often used depth budget.

Directors and stereographers can take advantage of DSP by recording actors movements, then camera movements, then stereoscopic parameters and review the perceived depth effects immediately on all targeted screen sizes. Our implementation of DSP has been tested by professional film-makers on an actual stereoscopic short film: Endless Night (see Sec. 5).

\section{RELATED WORK}

The difficulty of planning 3D cinematography has motivated research in the related areas of 3D calculators, 3D previz tools and onset 3D production tools (sometimes called durviz tools).

In the area of 3D production tools, we find 3ality Technica's "Stereo Image Processor", Franhaufer's "Stereoscopic Analyzer" (STAN) described in [15], Binocle's "TaggerMovie" and "TaggerLive" and the "Computational Stereo Camera System with Programmable Control Loop" from Heinzle et al.[4].

Those products allow the stereographer to have on-set feedback on how their acquisition images will look like in the projection room. The main constraint of those tools is the need of actual acquisition equipment: they require an actual 3-D rig complete with cameras, actors, and a full-size movie set.Under those circumstances, the test of exploratory shots becomes quickly expensive and time consuming. Cinematography students with novel ideas cannot "see their movie before shooting it" as proposed by Proferes[11].
The goal of a $3 D$ calculator is to help the stereographer to configure its rig (mainly the interaxial parameter), by defining the acquisition and the movie theater parameters. Those simulators allow to specify static depth limits in both worlds, namely near and far planes. The software will compute the interaxial parameter creating a 3D transformation between the scene and the movie theater, so that objects in the shooting near plane (btw. far plane) appear in the movie theater at the specified near plane (btw. far plane). As of today, more than 20 different $3 D$ calculators are available on the web ${ }^{1}$ The pioneers in this type of application were Inition with it's StereoBrain ${ }^{2}$ and FrameForge with its RealD Professional Stereo3D Calculator ${ }^{3}$ Only the Cine3D Stereographer $r^{4}$ claims to have a real-time simulation, allowing to simultaneously display the perceived depth as the filming parameters are interactively changed by the user.

Although tools have proven to be very helpful to the community, they all share two important limitations. The first important limitation of those approaches is its static nature. Actual shots are dynamic: actors, as well as sets and camera positions can (and will) move inside one shot. None of the above tools are designed to handle different settings changing across time. Each configuration has to be tested individually and they can not be animated over time. The second limitation is that the calculators only allow to define the volume where the action will take place. However, 3D filming involves not only the volume, but the composition and arrangement of the elements in this volume. Those tools do not allow to set different objects on the acquisition space, and see their relative position and deformation as they would be perceived in the projection room.

In the area of 3D previz, a virtual environment allows to create simple computer-generated shots. Among existing previz tools, we can find different approaches depending on the target user. A first approach focuses in the ease of use of the creation of the 3D world, by providing big databases of presets for actors, decors, and cameras. FrameForge Previz studio[12] with its Stereo Edition is a commercially available previz tool allowing to easily plan stereoscopic shots [5]. This environment is also able to handle dynamic shots. The user can configure the scene at different keyframes and the program will automatically create the animations. However, in this tool it is not possible to control the camera movements by interactively changing their settings with a physical device, like for example the Shoot Cut \& Play Camera[3]. In A Viewer-Centric Editor for Stereoscopic Cinema [7] techniques for stereoscopic shot planning and post-production are provided. Their method requires rough takes of the scene

${ }^{1}$ http://www.stereo-3d-info.de/3d-calculator.html

2 http://www.inition.co.uk/opinion/

stereobrain-calculator

ihttp://www. frameforge3d.com/Products /

iphone-App/

4 http://www.stereographer.ch/

StereographerSiteEN/index.php 


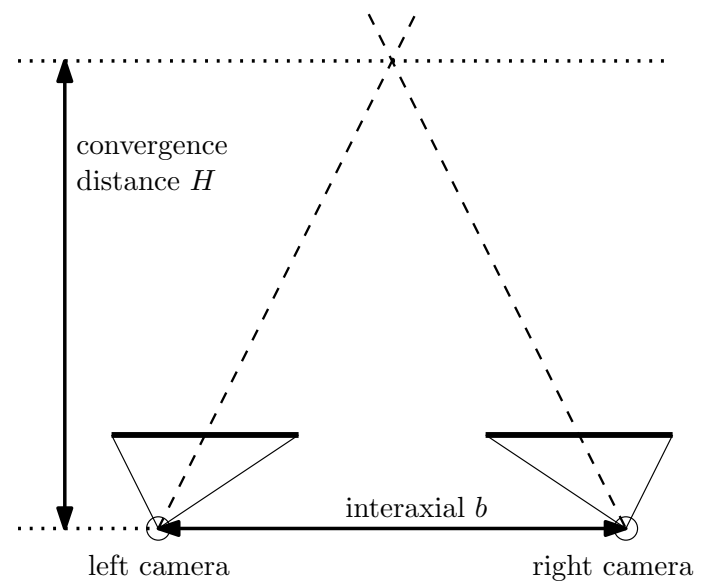

Fig. 1. Top view of a stereoscopic setting showing two parameters: interaxial and convergence distance

and/or still images. In contrast, we use 3D models of the scene. Their editor features a bird's eye view of the scene showing the perceived screen edge depths and the proscenium arch. In contrast, we offer a simulated view of the scene as perceived in the projection room.

Another related work is the Mixed Reality Pre-visualization tool for Filmmaking presented in [6]. They aim the production of shots composed of virtual characters on a real set, which are very challenging for the camera operator. With real-time tracking techniques they align the virtual action of the actors on the acquired images. This allows the camera operator to rehearse the camera movements that would best fit the animation. In its stereoscopic extension of the paper [10] they automatically set the rig parameters, interaxial and convergence, with the 1/30th rule, in order to be in the safe zone. An obvious limitation of both methods for our purpose is that they require a full-size studio during rehearsals.

None of the previously cited previz tools provides a visualization of how the shot would look like in the projection room.

\section{REAL TIME CAMERA CONTROL}

DSP consists of python scripts and OpenGL shaders running in the Blender Game Engine, which is multiplatform (Linux, Windows and OsX), and makes it easy to create 3D models for the sets, characters and props required in a movie previsualization. Moreover, a large online community provides free access to a large database of online models and animations. An additional benefit of the Blender Game Engine is that a large majority of USB devices, e.g., commercially available focus and aperture pullers, can be connected with the real-time OSC protocol [14]. In our implementation we used a USB game-pad controller.

\subsection{Stereoscopic controls}

DSP lets users take control of all camera parameters, including the two stereoscopic controls of professional rigs : interaxial, noted as $b$ and convergence distance, noted as $H$ (see Fig. 11. We assume a perfectly calibrated rig, with the segment joining both optical centers (baseline) being parallel to the projection planes. We consider that the convergence is done by shifting the optical centers of the cameras along their baseline. By doing so, keystone artefacts are avoided and zero disparity (corresponding to screen depth) can be set at any distance.

\subsection{Multitrack recording}

We provide a multitrack recording feature allowing to sequentially record the different parameters of a stereoscopic camera rig. Users can choose different roles to take control of different parameters. In the camera operator role, the user controls pan, title and zoom parameters. In the stereographer role, the user controls interaxial and convergence. In the Dolly assistant role, the user controls camera translation, etc.

Multi-track recording allows to replay previously recorded tracks while recording novel tracks controlled by one or more users taking different roles. Once they are recorded, the user can switch to another role and control its corresponding parameters. The time-line allows to navigate temporally and display the values of all the parameters at each instant. At any time the user can record again any track, exploring new possibilities and configurations. Previous recordings can be kept for comparison.

An example with a panning shot is shown in Fig. 22: Given an actor's movement in the set (a woman enters the room), the user can first select the Camera Operator role to control the camera position and follow the movement of the actress. The user can train and record its movements several times. Once the framing is satisfactory the user switches to Stereographer role, to control the Interaxial and the Convergence distance. All these parameters can, as in an actual shooting, be changed dynamically during the scene. The recording order of the parameters is not constrained. The user can explore the possibilities at will. Moreover, at any moment, the user is able to record again any track.

\section{VIRTUAL PROJECTION ROOM}

One of the main difficulties when shooting stereoscopic footage is the fact that the result depends on the geometry of the projection room. The artist needs to decide for which target screen size the images will be shot.

\subsection{Parameters}

We give the possibility to the artist to configure the virtual projection room by setting the following parameters. Screen 

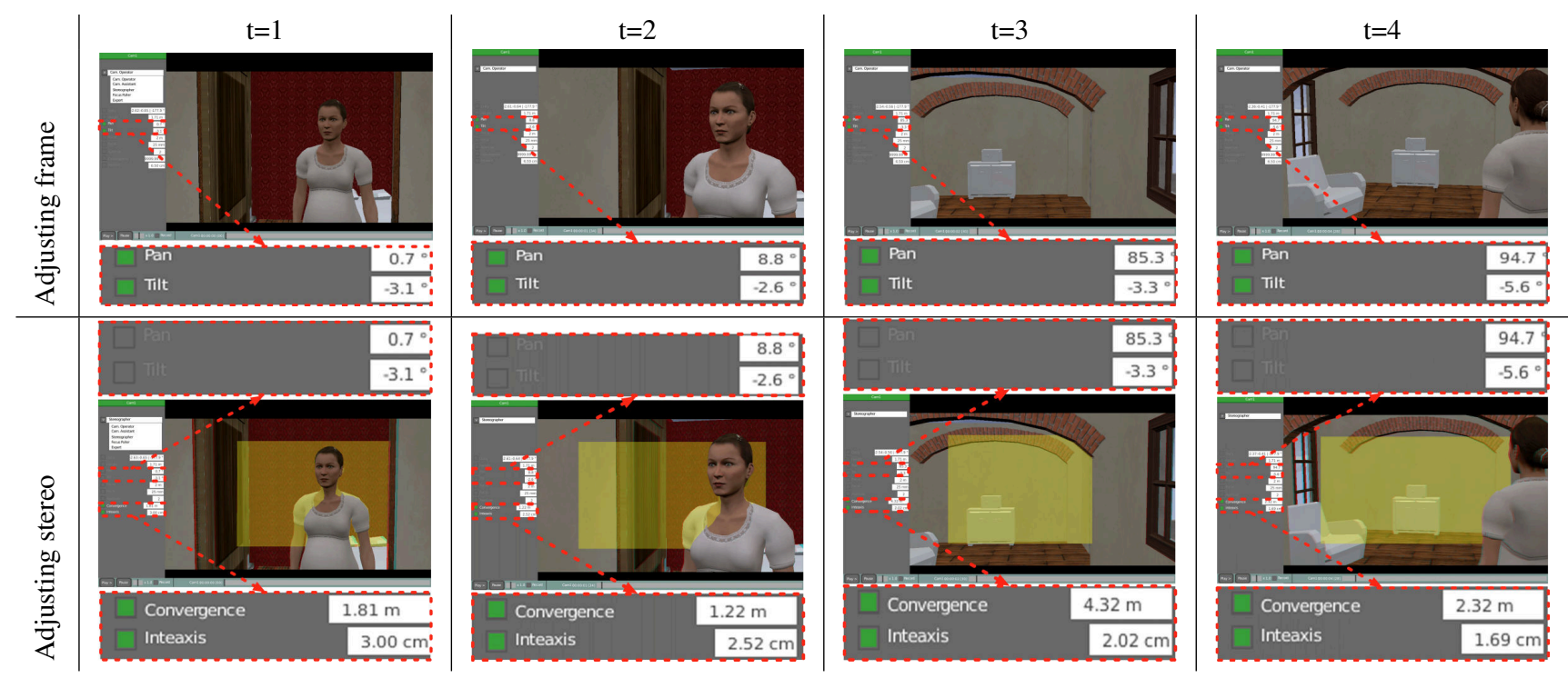

Fig. 2. First row (Adjusting frame): the user in Camera Operator role, controls the frame of the camera interactively, and follows the woman as she enters the room. The parameters of the camera, Pan and Tilt, are recorded (marked with a green box). Second row (Adjusting stereo): once the frame has been recorded, the user switches to Stereographer role and controls the interaxial and convergence distance interactively. The parameters of the camera, Pan and Tilt, are replayed (marked with a gray box) while Convergence and Interaxial are recorded (marked with a green box). The yellow semitransparent rectangle in the images shows the stereoscopic convergence plane in the scene.

size is the width of the target screen. Spectator position is the position of the spectator in the projection room. Near plane alert is a depth. Any object in front of the near plane will raise an alert. Far plane alert is a depth. Any object behind the far plane will raise an alert. The user can freely move it in the projection room, in order to see the deformations of the projected scene. See Fig. 4 to 8 for examples.

\subsection{Transformation}

Given the shooting configuration and the projection configuration, previous work [2] has shown that a non-linear geometric 3D transformation exists from the 3D world into the projection room. This transformation is complex for most common configurations, making it difficult for the director to see how the 3D shot will look like in the projection room.

Using the notation detailed in Fig. 3 the relationship between the true depth in the 3D scene and the perceived depth in the projection room can be written as:

$$
Z^{\prime}=\frac{H^{\prime}}{1-\frac{W^{\prime}}{b^{\prime}}\left(\frac{b}{W} \frac{Z-H}{Z}\right)} .
$$

The proposed virtual projection room relies on a purely geometric transformation. It doesn't take into account other well known 2D depth cues, like for example Light and shade or the Relative size of objects (smaller objects are farther away). These factors are well know to encode the depth in a 2D representation and are not taken into account in this work. More- over, some 3D deformations of the scene are acceptable when perceived by the spectator [8]. Despite its limitations, the virtual projection room provides vital information about the $3 \mathrm{D}$ deformations created by the current shooting and projection configurations.

\subsection{Simulation}

In our DSP tool we propose a virtual projection room simulation. It allows seeing at a very early stage, how the scene will look like when projected on the target screen.

We explain the computation with a simple example. While we are acquiring the scene presented in Fig. 4) (a woman and two spheres), the operator can set the interaxial and the convergence distance at will. We draw the corresponding convergence window as shown in Fig. 4 This window depends on the focal of the camera and the convergence distance. In Fig. 5 we show different views of the virtual projection room. They present the 3D perception of the spectator of the acquired images. For the first configuration we selected the dimensions of the projection room to exactly match those of the acquisition set. With this configuration $\left(b=b^{\prime} ; H=\right.$ $H^{\prime} ; W=W^{\prime}$ ), the eq. 1 becomes $Z^{\prime}=Z$. No deformations of the space are introduced.

Let us change the virtual projection room configuration by making the screen bigger $(10 \mathrm{~m})$ and moving the spectator to a viewing distance of $10 \mathrm{~m}$. Now the perceived 3D scene reveals important deformations, as shown in Fig. 6 Notice the complex deformation arising in the perceived depth in- 

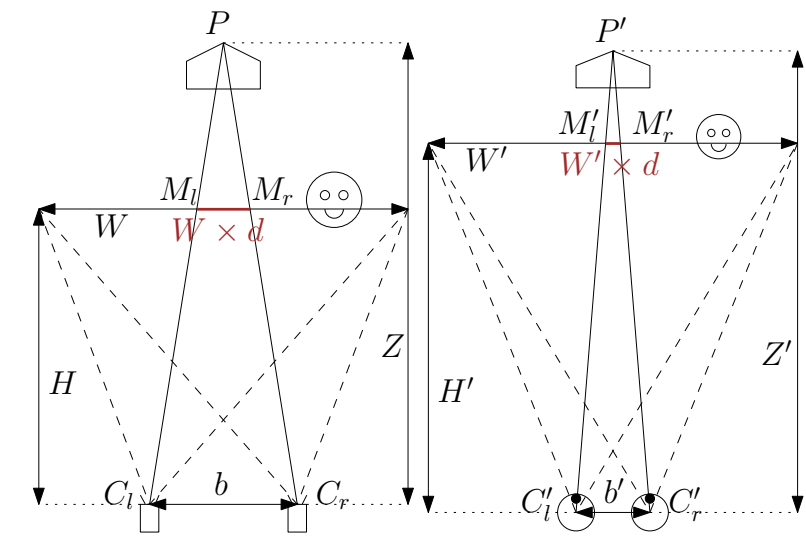

\begin{tabular}{|c|c|c|}
\hline Symbol & Shooting & Movie Theater \\
\hline \hline $\mathbf{C}_{l}, \mathbf{C}_{r}$ & camera optical center & eye optical center \\
\hline $\mathbf{P}$ & physical point of the scene & perceived 3D point \\
\hline $\mathbf{M}_{l}, \mathbf{M}_{r}$ & image points of $\mathbf{P}$ & screen points \\
\hline$b$ & interaxial & humain eye distance \\
\hline$H$ & convergence distance & screen distance \\
\hline$W$ & convergence plane size & screen size \\
\hline$Z$ & real depth & perceived depth \\
\hline$d$ & left-right disparity (as a fraction of $W$ ) \\
\hline
\end{tabular}

Fig. 3. Parameters describing the shooting geometry and the movie theater configuration (reproduced from [2].

troduced only by changing the screen size and the spectator position. We would like to draw the reader's attention to compare the right images on Fig. 5 and Fig. 6 . In the first, the screen is $2.5 \mathrm{~m}$ wide, and in the second it is $10 \mathrm{~m}$ wide, as shown by the smaller size of the spectator. Once the operator perceives the problem, he can change the acquisition parameters in order to obtain the desired result. In this case, by only decreasing the interaxial parameter, the perceived 3D can be improved as shown in Fig. 77 The user can, at any moment, freely move the viewpoints in the acquisition scene and the virtual projection room, as shown in Fig. 8 This ability allows the user to explore and better understand the perceived 3D scene.

In this example we chose to minimize 3D deformation in the projection room. In other cases, the deformation of the scene can be a narrative tool for the director. With this simple example we show the power of the feedback provided by the virtual projection room, which allows the director to quickly understand the complex 3D transformations involved with the projection and interactively adjust the acquisition parameters.

\section{EXPERIMENTAL RESULTS}

We tested our DSP tool during the shooting of a short stereoscopic movie. This short movie (Endless Night) takes place in an apartment, which we re-created in Blender 3D. Based on the director's storyboards, we created previz animations

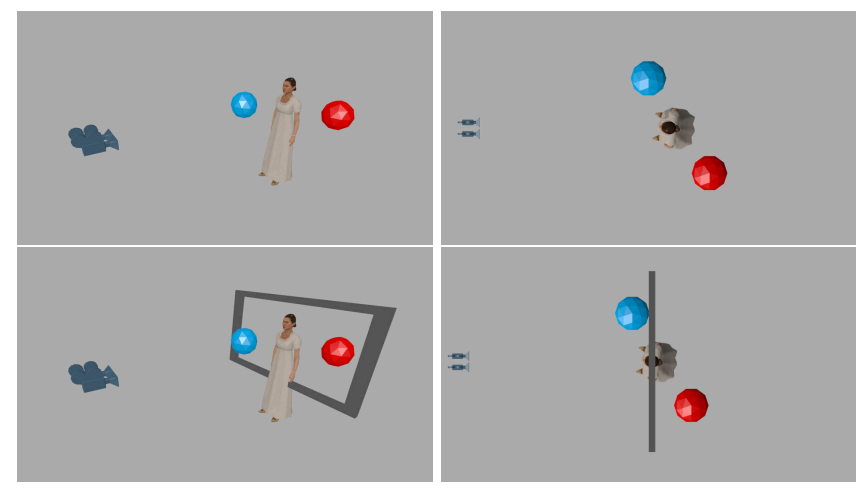

Fig. 4. Acquisition view of the Toy Scene. Left: perspective view. Right: top orthogonal view. The woman is at $2.5 \mathrm{~m}$ of the camera, and the woman's shoulders are $0.5 \mathrm{~m}$ wide. The spheres have a diameter of $0.5 \mathrm{~m}$. The blue one is $0.5 \mathrm{~m}$ in front of the woman, and the red one $1 \mathrm{~m}$ behind. Bottom row: the interaxial is set to $6.5 \mathrm{~cm}$. The convergence distance is set at $2.5 \mathrm{~m}$ of the camera (the depth of the woman). A window is displayed to help the operator validate the parameters.
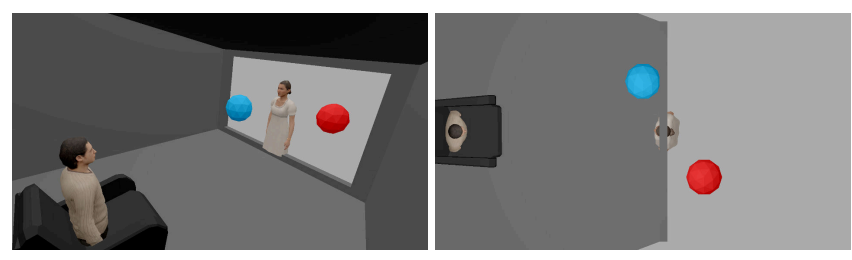

Fig. 5. Visualization of the virtual projection room for the images acquired with the configuration of Fig. 4. The interocular between the eyes of the spectator is $6.5 \mathrm{~cm}$, the screen is $2.5 \mathrm{~m}$ wide and the spectator is centered in the room at a $2.5 \mathrm{~m}$ distance of the screen. Because the virtual projection room configuration matches the acquisition configuration from Fig. 4. no 3D deformation is introduced. The 3D transformation is the Identity transformation. Left: perspective view. Right: top orthogonal view.

for ten shots of the movie, two of which are presented in this section, and in the supplementary videos.

DSP takes as input an annotated storyboard such as Fig. 9 and 10 . For each shot the storyboard provides the first and the last frames, together with floor-plan view drawings of the desired camera and actors movements and a written annotation on the desired mise-en-scene (shallow or deep shot, in front or at the back of the screen).

\subsection{Panning \& Dolly Shots}

In order to show the difficulty of the stereoscopic parameter setting, we focus on two dynamic shots: a panning shot and a dolly shot. In the panning shot the actress comes out from the room and enters the hall. The storyboard drawings are shown in Fig. 9. In the Dolly shot the woman simply walks 

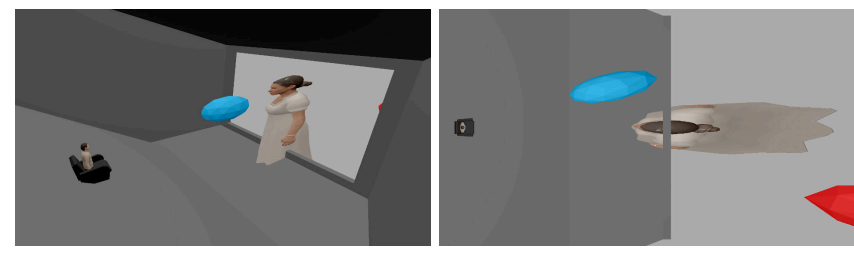

Fig. 6. Visualization of the virtual projection room for the images acquired with the configuration of Fig. 4. The interocular between the eyes of the spectator is $6.5 \mathrm{~cm}$, the screen is $10 \mathrm{~m}$ wide and the spectator is centered in the room at a $10 \mathrm{~m}$ distance of the screen. Important deformations of the perceived 3D scene are introduced. Left: perspective view. Right: top orthogonal view.
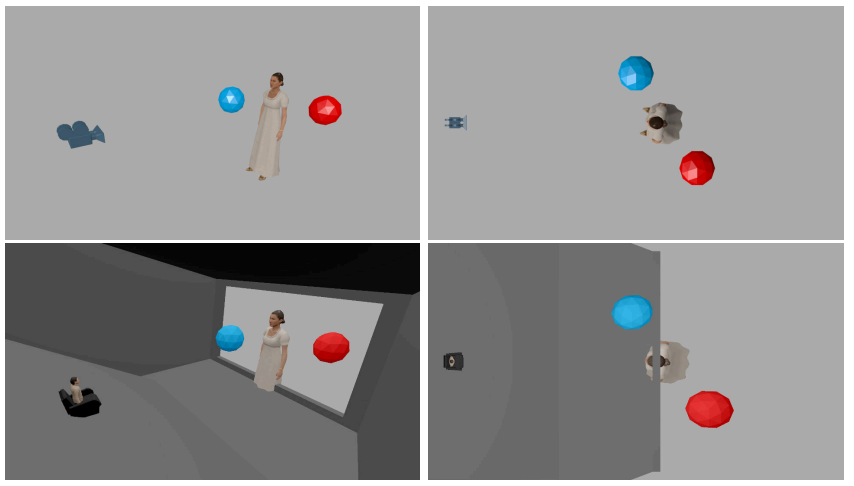

Fig. 7. First Row (Acquisition of the Toy Scene ): the interaxial is set to $2 \mathrm{~cm}$. The convergence distance is set at $2.5 \mathrm{~m}$ of the camera (the depth of the woman). Second Row (Visualization of the virtual projection room): The interocular between the eyes of the spectator is $6.5 \mathrm{~cm}$, the screen is $10 \mathrm{~m}$ wide and the spectator is centered in the room at a $10 \mathrm{~m}$ distance of the screen. The modification in the acquisition device allows to avoid the important 3D deformations of Fig. 6 .
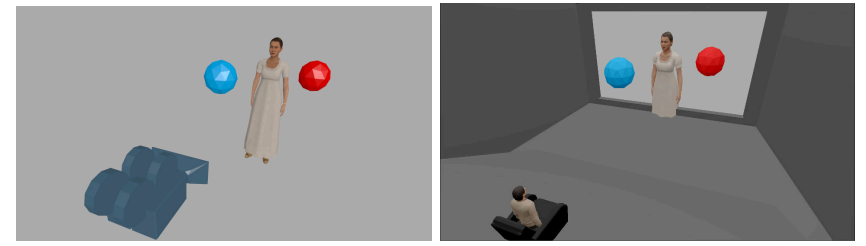

Fig. 8. A different viewpoint of the acquisition (left) and virtual projection room (right) corresponding to the configurations of Fig. 7
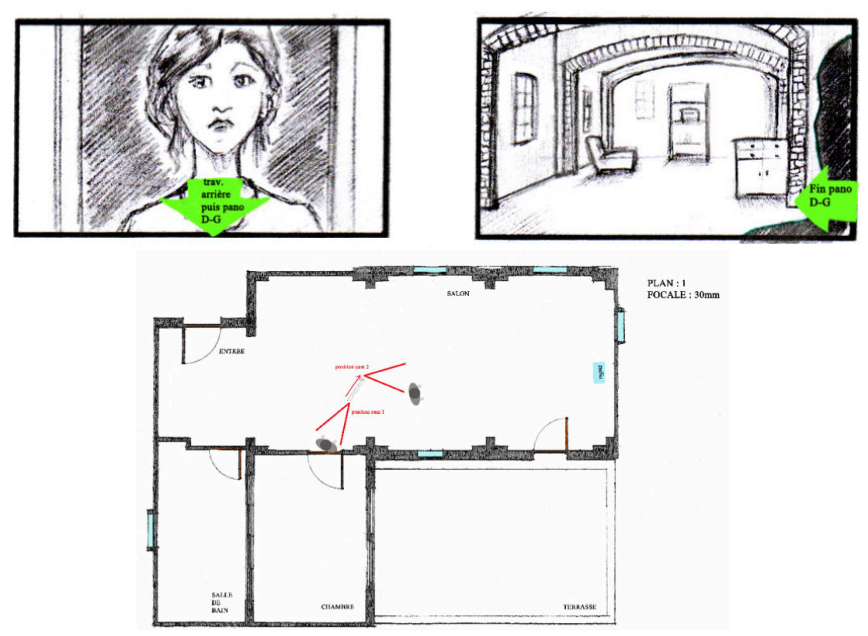

Fig. 9. Traditional storyboards are useful for placing actors and cameras, but provide little support for stereoscopic 3-D. We use them as input for previz. Top: Storyboard input for a panning shot. Bottom: Floor-plan view with director's annotations showing camera and actors displacements.

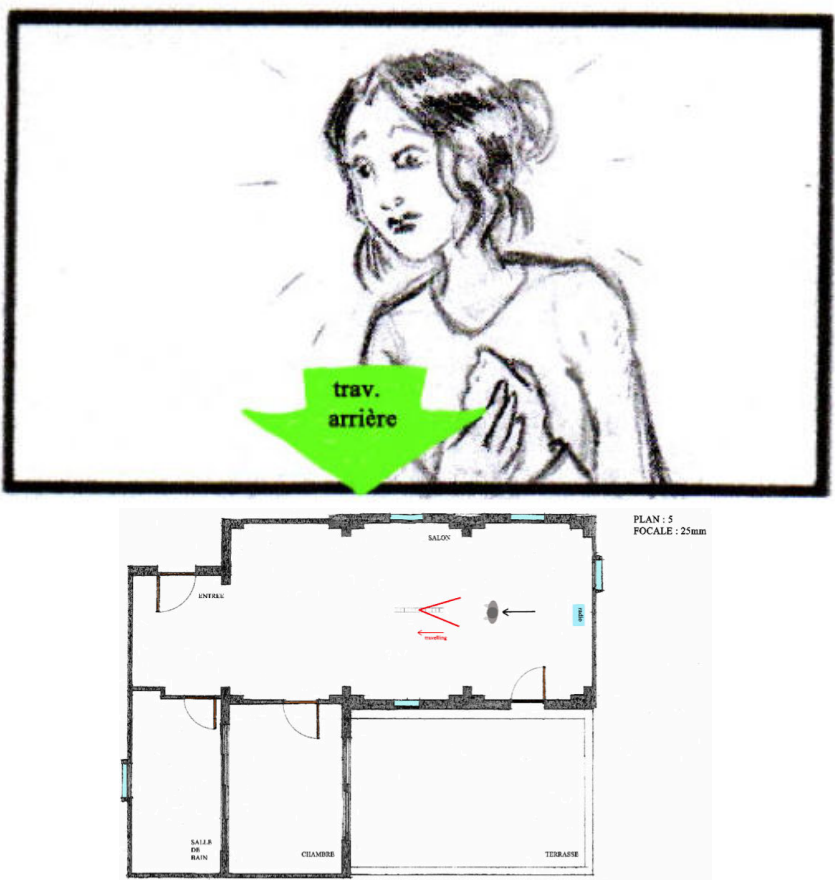

Fig. 10. Top: Storyboard input for a Dolly shot. Bottom: Floor-plan view with director's annotation showing camera and actors displacements. 
across the hall and the camera moves backwards. Its storyboard drawings are shown in Fig. 10.

Those shots are challenging because the motion of the camera introduces important changes on the acquired scene volume. The movement of the female actor also gives multiple choices to set the convergence distance. Several stereoscopic choices are possible depending on the desired 3D effect. DSP allows to play with different stereoscopic configurations, by dynamically changing the convergence distance and the interaxial parameters. In Fig. 11 and 12 we show the action recorded by DSP, as well as the actual rushes from a test shooting of the scene.

\subsection{Augmented storyboards}

Traditional storyboards such as Figs. 9 and 10 cannot be used to convey stereoscopic depth effects and mise-en-scene, except for a written description of the expected 3D. After previz, we can enrich this description with a top view of the virtual projection room (see Sec. 4). With this visual information, the director can directly convey the intended effect of the shot to the stereographer and other technical staff (cinematographer and camera operators).

\section{CONCLUSION}

We have presented a novel method for simulating the projection of a stereoscopic movie in a virtual previz environment. Together with real-time interaction techniques, our method overcomes several limitations of existing pre-production tools for stereoscopic movie-making. Most importantly, it makes it possible to visually convey the $3 \mathrm{D}$ deformations produced in a 3D projection in real time. Dynamic stereoscopic previz can be used to quickly preview complex stereoscopic 3-D shots, and to produce storyboards augmented with virtual projection room snapshots.

\section{ACKNOWLEDGEMENTS}

This work was funded by the French Government and the Caisse des dépôts et Consignations as part the ACTION3DS research project. We than Jonathan Bocquet and the entire "Endless Night" production team at Binocle 3D and Ecole Nationale Supérieure Louis Lumière for letting us reproduce their pre-production work.

\section{REFERENCES}

[1] Laurent Chauvier, Kevin Murray, Simon Parnall, Ray Taylor, and J Walker. Does size matter? the impact of screen size on stereoscopic $3 \mathrm{dtv}$. In IBC conference, 2010 .

[2] Frédéric Devernay and Paul Beardsley. Stereoscopic cinema. In Rémi Ronfard and Gabriel Taubin, editors,
Image and Geometry Processing for 3-D Cinematography, pages 11-51. Springer Berlin Heidelberg, 2010.

[3] Xavier Gouchet, Remi Quittard, and Nicolas Serikoff. Scp camera. Proc. of SIGGRAPH emerging technologies, page 16, 2007.

[4] Simon Heinzle, Pierre Greisen, David Gallup, Christine Chen, Daniel Saner, Aljoscha Smolic, Andreas Burg, Wojciech Matusik, and Markus Gross. Computational stereo camera system with programmable control loop. Transaction on Graphics, 30:94:1-94:10, August 2011.

[5] Jay. Holben. Frameforge 3d studio simplifies storyboard process. American Cinematographer Magazine, pages 101-106, October 2003.

[6] Ryosuke Ichikari, Ryuhei Tenmoku, Fumihisa Shibata, Toshikazu Ohshima, and Hideyuki Tamura. Mixed reality pre-visualization for filmmaking: On-set camerawork authoring and action rehearsal. Int. J. Virtual Reality, 7(4):25-32, 2008.

[7] S.J. Koppal, C.L. Zitnick, M. Cohen, Sing Bing Kang, B. Ressler, and A. Colburn. A viewer-centric editor for 3d movies. Computer Graphics and Applications, IEEE, 31(1):20 -35, jan.-feb. 2011.

[8] Bernard Mendiburu. 3D Movie Making: Stereoscopic Digital Cinema from Script to Screen. Focal press, 2009.

[9] Bernard Mendiburu. 3d TV and 3d cinema: tools and processes for creative stereoscopy. Focal press, 2011.

[10] Shohei Mori, Fumihisa Shibata, Asako Kimura, and Hideyuki Tamura. Stereo camera tracking for mixed reality-based previz of stereoscopic $3 \mathrm{~d}$ cinema using icp algorithm. Proceedings of International Conference on Machine Vision Applications, 2013.

[11] Nicholas T Proferes. Film Directing Fundamentals: see your film before shooting. Focal press, 2008.

[12] Ken Schafer. Frameforge previz studio. http: / / www. frameforge3d. com.

[13] Kazuhiko Ukai and Peter A Howarth. Visual fatigue caused by viewing stereoscopic motion images: Background, theories, and observations. Displays, 29(2):106-116, 2008.

[14] M. Wright and A. Freed. Open sound control: A new protocol for communicating with sound synthesizers. In International Computer Music Conference, 1997.

[15] F Zilly, M Muller, P Kauff, and R Schafer. Stan - an assistance system for $3 \mathrm{~d}$ productions: from bad stereo to good stereo. Proc. of ITG Conference on Electronic Media Technology, pages 1-6, 2011. 
$\mathrm{t}=1$

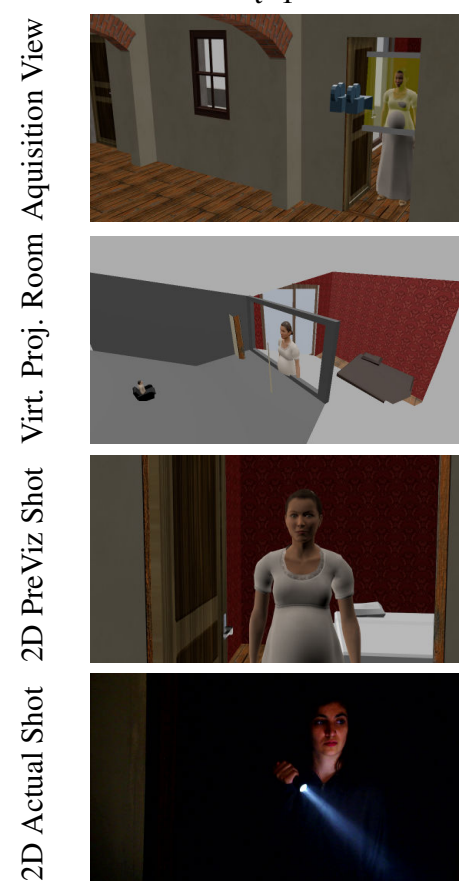

$\mathrm{t}=2$
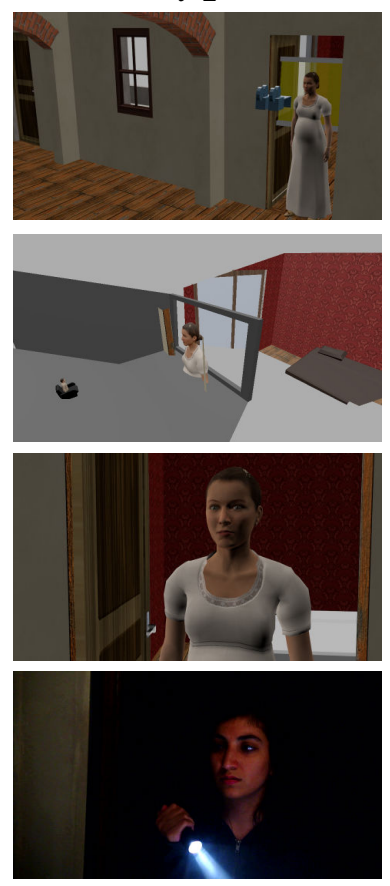

$\mathrm{t}=3$
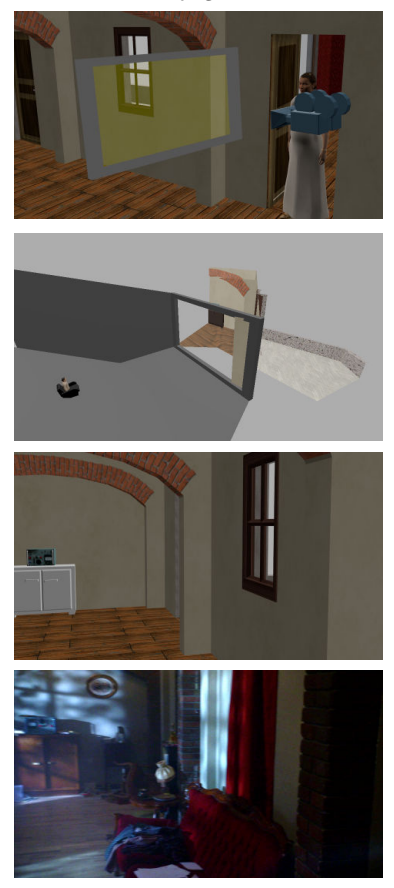

$\mathrm{t}=4$
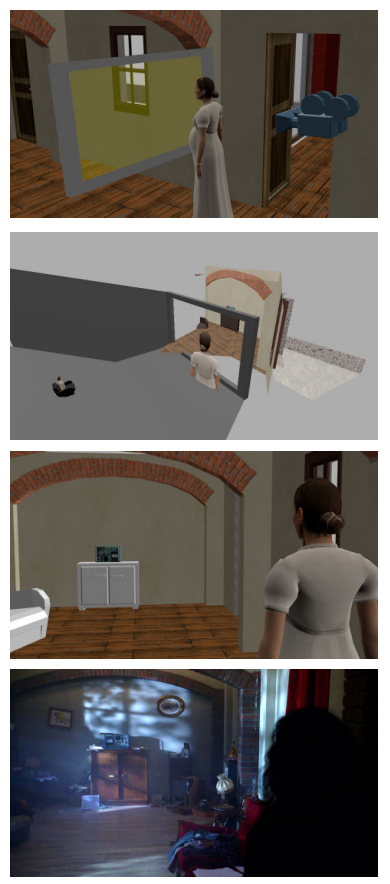

Fig. 11. Previz results for a panning shot. Columns show different times in the shot, arranged chronologically from left to right. First row: shooting set; second row: projection room; third row: previz results; bottom row: actual rushes. The stereoscopic parameters are adjusted across the shot to adjust the stereoscopic effect.

$\mathrm{t}=1$

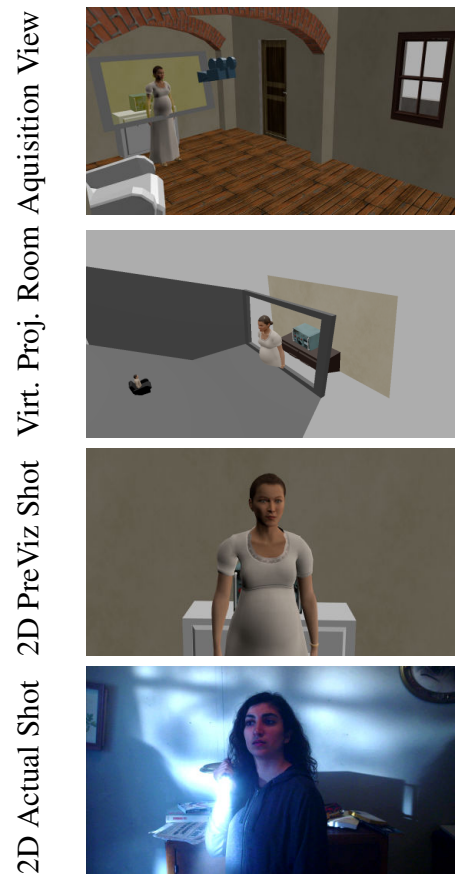

$\mathrm{t}=2$
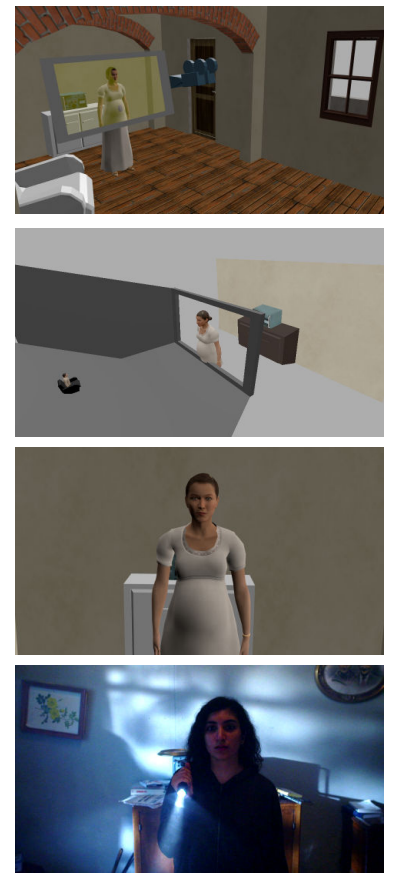

$t=3$
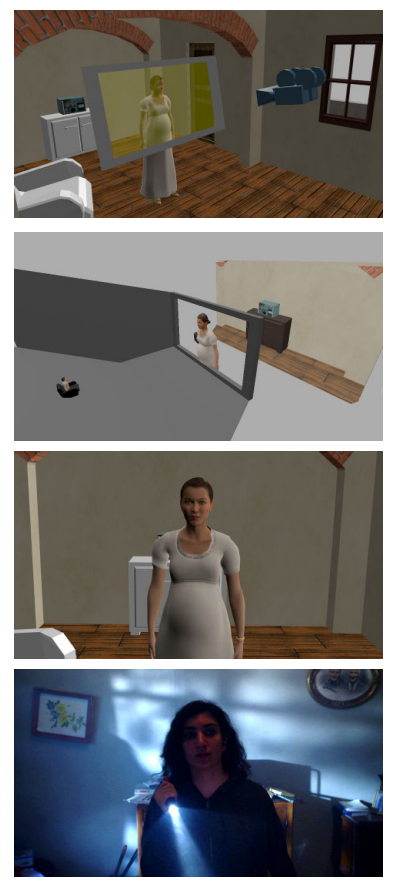

$\mathrm{t}=4$
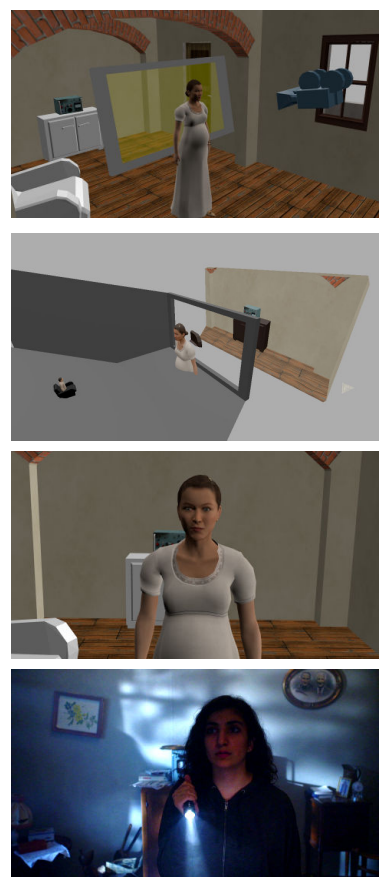

Fig. 12. Previz results for a Dolly shot. The actor moves away from the furniture across the room. The difficulty of this shot is to handle the increasing volume of the scene, as the actor moves farther away from the back wall. Moreover, the distance of the actor to the camera decreases as she walks. 